result from changes in an inward or an outward component. Although it is relatively easy to establish that the inward current is carried by $\mathrm{Na}^{+}$or $\mathrm{Ca}^{2+}$ ions by removing them from the external solution, it is difficult to show directly that $\mathrm{K}^{+}$ions carry the outward current. An elegant way of doing this was recently reported by Neher and Lux (J. gen. Physiol., 61, 385; 1973). They used a $\mathrm{K}^{+}$-sensitive microelectrode to measure changes in $\mathrm{K}^{+}$outside snail neurones, and showed that the total amount of $\mathrm{K}^{+}$released by the cell during a clamp pulse was in good agreement with that calculated assuming that the outward current was carried only by $\mathrm{K}^{+}$ions. Lux and Eckert (see page 574 of this issue of Nature) have now applied this ingenious technique to a study of apparent $\mathrm{K}$ inactivation. When the outward current was reduced by procedures expected to produce $\mathrm{K}$ inactivation, the actual release of $\mathrm{K}^{+}$, as determined by the $\mathrm{K}^{+}$-sensitive microelectrodes, was not equally reduced. They conclude that there must be a slow inward current as well as the normal slow outward current. Part of the apparent $\mathrm{K}$ inactivation was the result of this unsuspected inward current, which would make the outward current as recorded by the clamp smaller than the true outward current. The ion or ions carrying this slow inward current are so far unidentified, but are presumably $\mathrm{Na}$ or Ca.

ROger C. THOMAS

L. Donald Partridge

\section{Germline antibody genes}

THE genetic basis for antibody diversity is a subject of continuing interest though the argument between germline and somatic models of antibody diversity has abated recently. The large number of different amino acid sequences so far determined for $V$ regions of mouse Kappa chains, point to there being at least a hundred $V_{\text {Kappa }}$ genes and there is probably a similar minimum number of $V_{\text {II }}$ genes. The question as to whether somatic processes are used to increase the diversity encoded in the multiple germline $V$ genes remains open. Now the major effort is aimed at defining the way in which the $V$ and $C$ genes are arranged in a chromosome and the way in which they function.

At present it is thought that the genes which code for $\mathrm{H}$ chains $\left(V_{\mathrm{H}}\right)$ are present on the same chromosome with the set of about ten $C_{\mathrm{H}}$ genes, one $C_{\mathrm{H}}$ gene for each class of antibody. In this model any $V_{\mathrm{H}}$ gene can be paired with any $C_{H}$ gene to give a gene pair coding for a single heavy chain. Although there is considerable evidence to support this scheme, at present it is only an outline of what must be a fascinating genetic process. The $C$ genes for both light and heavy chains map as single genes in a Mendelian fashion. Similar mapping of individual $V$ genes has been limited by the lack of suitable phenotypic markers. Recently individual antibodies have been characterised by either their idiotype (characteristic antigenic determinants defined by antisera raised against the antibody under study) fine specificity (definition of an antibody combining site by the quantitation of cross reactions) or spectrotype (characteristic isoelectric focusing spectrum of an antibody). Using these genetic markers six $V_{\mathrm{H}}$ genes have been identified in the mouse and a search is now being made for recombinants so that a map of the $V_{\mathrm{H}}$ region of the chromosome can be drawn. Each of these $V_{\mathrm{H}}$ genes, in agreement with the model I have mentioned, has been found to be closely linked to the $C_{\mathrm{H}}$ loci.

Eichmann, Tung and Nisonoff reponted in last week's edition of Nature $(\mathbf{2 5 0}, 509-511 ; 1974)$ strong evidence for a recombinational event separating two of the known $V_{\mathrm{H}}$ genes $A R S$ and $A 5 A$. These genes had been identified by the idiotype present on $\mathbf{A} / \mathbf{J}$ strain mouse antibodies directed against $p$-azophenylarsonate (ars) and group A streptococcal carbohydrate respectively. A/J mice can make a variety of different antibodies directed against each of these two antigens. The initial response of $\mathrm{A} / \mathrm{J}$ mice to ars always includes an antibody (or a family of antibodies) carrying the ARS idiotypic determinant. The A5A idiotype has been found to be associated with a particular antibody spectrotype and cells producing that antibody have been cloned in vivo (Eur. J. Immun., 2, 301; 1972). The inheritance of $A 5 A$ in the $C_{\mathrm{H}}$ linkage group was shown by Eichmann and Berek (Euro. J. Immunol., 3, 599; 1973) who mated $\mathrm{A} / \mathrm{J}$ mice against $\mathrm{BALB} / \mathrm{c}$ mice, a strain which efficiently makes antibody against group A carbohydrate but has never shown that $A 5 A$ idiotype; the $F_{1}$ progeny were backcrossed against BALB/c. Of the backcross offspring 16/29 were homozygous for the BALB/c $C_{\mathrm{H}}$ genes; all but one of those 16 were, as expected, negative for the A5A idiotype on their anti-group A carbohydrate antibody. The odd mouse $\mathrm{BB} \delta^{7} 7$ showed a recombinant phentotype with the $A 5 A$ gene apparently present in the BALB/c $C_{\mathrm{H}}$ linkage group.

In Eichmann et al.'s latest study, BB $0^{7} 7$ has been again backcrossed against $\mathrm{BALB} / \mathrm{c}$ and the progeny support the idea that a new linkage of $A 5 A$ to the BALB/c $C_{\mathrm{H}}$ loci has occurred as the result of a recombinational event. Many of the progeny of $\mathrm{BB} \delta^{\top 7} 7$ have been tested for the ARS idiotype. In this respect the backcrosses behave just like the parental $\mathrm{BALB} / \mathrm{c}$ mice and make antiars lacking the ARS idiotype. The one recombinational event observed among the three genes under study is consistant with a simple linear map $A 5 A-\mathrm{X}-A R S-$ $C_{\mathrm{H}}$-where $\mathrm{X}$ shows the position of the crossover. Further crossovers are needed in order to estimate map distances between these three genes.

The occurrence of a recombinant in only sixteen progeny in which it could be detected is either a very fortuitous event or else it is indicative of a large map distance between $A 5 A$ and the other two genes. One map distance in the $H$ chain linkage group of the mouse was reported by Riblet at the workshop on immunoglobulin variable region genetics (Bethesda, Maryland, March 25 and 26, 1974). Riblet studied the $D E X$ gene which is characterised by an idiotypic specificity found on two BALB/c myeloma antibodies binding $\alpha-1,3$ dextran. Only two recombinational events were observed in a total of 530 crosses in which a recombination could have been detected. At the time of writing their article, Eichmann and his colleagues had screened 73 meaningful crosses and $\mathbf{B B} \sigma^{7} 7$ remains the only recombinant. The next recombinant is keenly anticipated since the measurement of the distance between $A 5 A$ and $A R S$ will be indicative of the number of $V$ genes which might lie between these markers.

Alan R. Williamson

\section{Reappearance of hexosaminidase A}

SINCE it became possible to fuse somatic cells using Sendai virus people have been investigating the complementation (supply of the other's needs by each parental cell) of closely similar genetic defects. Complementation, in hybrids and heterokaryons (the multinucleated cells produced by fusion) has provided useful information about recessive mutants, in cases, for example, where the precise metabolic lesion is not known and genetic heterogeneity has been neatly demonstrated (see, for example, Kao et al., Science, 164, 312; 1969; de Weerd-Kastelein et al., Nature 